CLINICAL STUDY

\title{
Circulating high-molecular-weight adiponectin is upregulated in preeclampsia and is related to insulin sensitivity and renal function
}

\author{
Mathias Fasshauer ${ }^{1,2}$, Theresa Waldeyer ${ }^{3}$, Jeannette Seeger ${ }^{1}$, Susanne Schrey ${ }^{3}$, Thomas Ebert ${ }^{1}$, Jürgen Kratzsch ${ }^{4}$, \\ Ulrike Lössner ${ }^{1}$, Matthias Blüher ${ }^{1}$, Michael Stumvoll ${ }^{1}$, Renaldo Faber ${ }^{3}$ and Holger Stepan ${ }^{3}$ \\ ${ }^{1}$ Department of Internal Medicine III, University of Leipzig, Ph.-Rosenthal-Str. 27, 04103 Leipzig, Germany, ${ }^{2}$ Interdisciplinary Center for Clinical \\ Research (IZKF) Leipzig, 04103 Leipzig, Germany, ${ }^{3}$ Department of Obstetrics and ${ }^{4}$ Institute of Laboratory Medicine, University of Leipzig, \\ 04103 Leipzig, Germany \\ (Correspondence should be addressed to M Fasshauer; Email: mathias.fasshauer@medizin.uni-leipzig.de)
}

\begin{abstract}
Objective: Preeclampsia (PE) is a serious cardiovascular complication in pregnancy which is associated with an increased future metabolic and cardiovascular risk for mother and newborn. Recently, a paradoxical upregulation of the insulin-sensitizing and anti-atherogenic adipokine adiponectin has been shown in PE. Furthermore, high-molecular-weight (HMW) adiponectin has been suggested as the biologically active form of this adipokine.

Design and methods: HMW adiponectin and total adiponectin serum concentrations were quantified by ELISA in PE $(n=16)$ patients and pregnant control women without PE $(n=20)$. Furthermore, HMW adiponectin and total adiponectin were correlated to clinical and biochemical measures of renal function, glucose, and lipid metabolism, as well as inflammation.

Results: Median maternal HMW adiponectin and total adiponectin levels were significantly and independently upregulated almost twofold in PE when compared with controls. HMW adiponectin and total adiponectin correlated positively with creatinine and negatively with fasting insulin in univariate and multivariate analyses.

Conclusions: We show that maternal HMW adiponectin and total adiponectin serum concentrations are significantly increased in PE and are positively associated with markers of insulin sensitivity and renal dysfunction. Adiponectin might be part of a physiological feedback mechanism improving insulin sensitivity and cardiovascular health in PE.
\end{abstract}

European Journal of Endocrinology 158 197-201

\section{Introduction}

Preeclampsia (PE), which is characterized by hypertension, proteinuria, and endothelial dysfunction, is a serious cardiovascular complication in pregnancy (1). Both mother and newborn have a significantly increased future risk for metabolic and cardiovascular diseases as a consequence of a preeclamptic pregnancy (1).

The pathogenesis of PE has been better elucidated in recent years. Thus, dysregulation of angiogenic and antiangiogenic factors including soluble feline McDonough sarcoma (fms)-like tyrosine kinase 1 and endoglin contribute to the disease (2-5). Furthermore, PE shares features of the metabolic syndrome (1) and recent studies suggest that adipocyte-secreted factors play an important role in the pathogenesis of this pregnancy complication. Among these so-called adipokines, increased concentrations of the appetite-suppressive, adipose tissue-derived factor leptin were found in PE (6). Interestingly, upregulation of leptin precedes the clinical onset of the disease and it has been suggested that hyperleptinemia is a compensatory response to increase nutrient delivery to the underperfused placenta $(6,7)$. Besides leptin, the proinflammatory adipokine tumour necrosis factor- $\alpha$ (TNF- $\alpha$ ) is increased about twofold in women with PE (8). Studies in pregnant rats showed convincingly that this extent of TNF- $\alpha$ upregulation is sufficient to increase mean arterial pressure by $27 \mathrm{mmHg}(9)$. Similar to TNF- $\alpha$, maternal IL-6 levels are significantly increased threefold in women with PE (8). Furthermore, circulating IL-6 increased from the first to the third trimester in women with $\mathrm{PE}$, but not in healthy pregnant controls (10). In contrast to TNF- $\alpha$ and IL-6, adiponectin is an insulin-sensitizing $(11,12)$ and anti-atherogenic adipokine (12-14), serum levels of which are decreased when features of the metabolic syndrome including obesity, dyslipidemia, and type 2 diabetes mellitus are present (15). Furthermore, epidemiological data suggest that low levels of adiponectin at baseline are associated with an increased risk to develop type 2 diabetes 
mellitus (15). In contrast, circulating adiponectin is not significantly associated with coronary heart disease in humans (16), despite the fact that this adipokine shows potent anti-atherogenic effects in various animal models (12-14). Interestingly, adiponectin circulates as a trimer (low molecular weight), hexamer (medium molecular weight), and high molecular weight (HMW) form (15) and HMW adiponectin has been postulated as the active form of the adipokine $(17,18)$. Since cardiometabolic risk is significantly increased in PE, it was hypothesized several years ago that circulating adiponectin might be decreased in this pregnancy-associated disease. However, a paradoxical upregulation of total adiponectin has been presented in various (19-23) but not all studies (24).

In contrast to total adiponectin, little is known about HMW adiponectin concentrations in PE. Furthermore, it has not been elucidated which factors predict HMW adiponectin in this pregnancy complication. Therefore, we determined HMW adiponectin levels in 20 pregnant controls and $16 \mathrm{PE}$ patients using a novel, specific ELISA (25). Furthermore, circulating HMW adiponectin was correlated with clinical and biochemical measures of renal function, glucose, and lipid metabolism, as well as inflammation.

\section{Subjects and methods}

\section{Subjects}

For this study, 16 pregnant women with PE and 20 gestational age-matched controls were recruited from the Department of Obstetrics, University of Leipzig. None of the women were in labor at the time of the blood sampling. $\mathrm{PE}$ was defined as gestational blood pressure elevation $>$ $140 \mathrm{mmHg}$ systolic or $>90 \mathrm{mmHg}$ diastolic, accompanied by proteinuria in women who were normotensive before 20 weeks of gestation, according to the Report of the National High Blood Pressure Education Program Working Group on High Blood Pressure in Pregnancy (26). Body mass index (BMI) was calculated as weight before pregnancy divided by squared height and the BMI of the study population ranged from 16.9 to $30.5 \mathrm{~kg} / \mathrm{m}^{2}$. The patients were between the ages of 18 and 40 years. Homeostasis model assessment of insulin resistance (HOMA-IR) was determined as described previously (27). Patients with generalized inflammation, renal diseases, and diabetes mellitus were excluded from the study. The protocol of the study was approved by the local ethics committee and all patients gave written informed consent before taking part.

\section{Assays}

A venous blood sample was obtained after an overnight fast, immediately separated by centrifugation at $4000 \boldsymbol{g}$ for $10 \mathrm{~min}$, and frozen at $-80{ }^{\circ} \mathrm{C}$. Serum insulin was measured with a two-site chemiluminescent enzyme immunometric assay for the Immulite Automated Analyzer (Diagnostic Products, Los Angeles, CA, USA). Circulating HMW adiponectin (Fujirebio, Tokyo, Japan) was determined according to the manufacturer's instructions. The HMW adiponectin ELISA detected adipokine in a range between 0.4 and $50 \mu \mathrm{g} / \mathrm{l}$. Serum samples were initially diluted 441 -fold as recommended by the manufacturer and further dilutions were performed when HMW adiponectin levels were above the detection range $(=50 \mu \mathrm{g} / \mathrm{l} \times 441=22.05 \mathrm{mg} / \mathrm{l})$. While the degree of precision of the HMW adiponectin ELISA system in terms of coefficient of variance (\%) of intra-assay was between 2.4 and $3.0 \%$, that of inter-assays was between 4.2 and $5.1 \%$ (25). Total adiponectin was determined with a commercial assay system from Mediagnost (Reutlingen, Germany) according to the manufacturer's instructions. Serum creatinine, glucose, free fatty acids (FFA), cholesterol, triglycerides, and C-reactive protein (CRP) were measured by standard laboratory methods in a certified laboratory.

\section{Statistical analysis}

SPSS software version 11.5 was used for all statistical analyses (SPSS, Chicago, IL, USA). Differences in circulating HMW and total adiponectin between control and PE patients were assessed by Mann-Whitney $U$ test. Correlations were performed using Spearman's rank correlation method. To adjust the effects of covariates and identify independent relationships, multivariate linear regression analyses were performed. Distribution was tested for normality using Shapiro-Wilk $W$ test and non-normally distributed parameters were logarithmically transformed before multivariate analyses. A $P$ value of $<0.05$ was considered statistically significant in all analyses.

\section{Results}

\section{Circulating HMW adiponectin serum levels are increased in PE patients when compared with controls}

The clinical characteristics of the subgroups studied (control, PE) are summarized in Table 1. All continuous variables are given as median \pm interquartile range. Maternal HMW adiponectin levels were significantly increased in subjects with PE $(16.03 \pm 12.53 \mathrm{mg} / \mathrm{l})$ when compared with healthy pregnant controls $(8.09 \pm$ $4.88 \mathrm{mg} / \mathrm{l} ; \mathrm{P}<0.01$; Table 1 ). This difference in circulating HMW adiponectin was also seen after adjustment for BMI $(P<0.01$; data not shown). Similarly, total adiponectin concentrations were higher in PE patients $(12.15 \pm 8.88 \mathrm{mg} / \mathrm{l})$ when compared with healthy pregnant controls $(6.75 \pm 3.55 \mathrm{mg} / \mathrm{l} ; \mathrm{P}<0.01$; Table 1$)$. PE patients were significantly older $(33.0 \pm 10.0$ years $)$ when compared with control subjects $(26.5 \pm 10.5$ years $)$. In contrast, BMI $\left(22.1 \pm 4.2\right.$ vs $\left.20.3 \pm 3.7 \mathrm{~kg} / \mathrm{m}^{2}\right)$ and 
Table 1 Baseline characteristics of the study population.

\begin{tabular}{lcc}
\hline & Control & PE \\
\hline$N$ & 20 & 16 \\
HMW adiponectin (mg/l) & $8.09 \pm 4.88$ & $16.03 \pm 12.53^{*}$ \\
Total adiponectin (mg/l) & $6.75 \pm 3.55$ & $12.15 \pm 8.88^{*}$ \\
Age (years) & $26.5 \pm 10.5$ & $33.0 \pm 10.0^{*}$ \\
BMI ( $\left(\mathrm{kg} / \mathrm{m}^{2}\right)$ & $20.3 \pm 3.7$ & $22.1 \pm 4.2$ \\
SBP (mmHg) & $105 \pm 30$ & $170 \pm 34^{*}$ \\
DBP (mmHg) & $70 \pm 14$ & $102 \pm 26^{*}$ \\
Gestational age at blood & $215 \pm 23$ & $200 \pm 47$ \\
sampling (days) & & \\
Creatinine ( $\mu \mathrm{mol} / \mathrm{l})$ & $54 \pm 17$ & $66 \pm 24^{*}$ \\
FG (mmol/l) & $3.59 \pm 0.58$ & $3.53 \pm 0.61$ \\
FI (pmol/l) & $62 \pm 34$ & $39 \pm 51$ \\
HOMA-IR & $1.39 \pm 0.88$ & $1.13 \pm 1.86$ \\
FFA (mmol/l) & $0.37 \pm 0.31$ & $0.89 \pm 0.45^{\star}$ \\
Cholesterol (mmol/l) & $6.75 \pm 1.98$ & $6.46 \pm 1.95$ \\
TG (mmol/l) & $2.59 \pm 1.89$ & $3.27 \pm 1.62$ \\
CRP (mg/l) & $1.65 \pm 2.10$ & $7.60 \pm 50.45^{\star}$ \\
\hline
\end{tabular}

$\mathrm{BMI}$, body mass index; $\mathrm{CRP}, \mathrm{C}$ reactive protein; $\mathrm{DBP}$, diastolic blood pressure; FFA, free fatty acids; FG, fasting glucose; FI, fasting insulin; HOMA-IR, Homeostasis model assessment of insulin resistance; SBP systolic blood pressure; TG, triglycerides. Values for median \pm interquartile range are shown. ${ }^{\star} P<0.05$ when compared with control as assessed by Mann-Whitney $U$ test.

gestational age at blood sampling $(200 \pm 47$ vs $215 \pm$ 23 days) were not significantly different in the two groups (Table 1). Systolic (SBP) and diastolic (DBP) blood pressure, serum creatinine, FFA, and CRP were significantly elevated in patients with $\mathrm{PE}$ when compared with controls (Table 1). In contrast, measures of insulin sensitivity (fasting glucose, fasting insulin, and HOMA-IR) and lipid metabolism (cholesterol and triglycerides) were not different between the two groups (Table 1).

\section{Univariate correlations}

When all subjects $(n=36)$ were analyzed, both serum HMW and total adiponectin levels correlated positively with creatinine and negatively with fasting insulin (Table 2). In addition, total adiponectin but not HMW adiponectin positively correlated with SBP and FFA (Table 2). In contrast, no correlation was found between HMW adiponectin and total adiponectin on one hand and age, BMI, DBP, fasting glucose, cholesterol, triglycerides, and CRP on the other hand (Table 2). Interestingly, a significant negative correlation between HMW adiponectin and fasting insulin could also be detected when the two subgroups (control, PE) were studied separately (controls: $r=-0.484$, $P=0.031$; PE: $r=-0.550, P=0.027)$. Furthermore, HMW adiponectin and total adiponectin serum levels were strongly and positively correlated (Table 2 ).

\section{Multivariate correlations}

In multiple regression analysis including all subjects $(n=36)$, the association between HMW adiponectin and total adiponectin serum concentrations, and fasting insulin and serum creatinine, remained significant when
Table 2 Univariate correlations with serum high-molecular-weight (HMW) adiponectin and total adiponectin concentrations in all subjects $(n=36)$.

\begin{tabular}{lcc}
\hline & $\begin{array}{c}\text { HMW adiponectin } \\
\boldsymbol{r} / \boldsymbol{P}\end{array}$ & $\begin{array}{c}\text { Total adiponectin } \\
\boldsymbol{r} / \boldsymbol{P}\end{array}$ \\
\hline Age (years) & $0.205 / \mathrm{NS}$ & $0.178 / \mathrm{NS}$ \\
$\mathrm{BMI}\left(\mathrm{kg} / \mathrm{m}^{2}\right)$ & $-0.009 / \mathrm{NS}$ & $0.095 / \mathrm{NS}$ \\
$\mathrm{SBP}(\mathrm{mmHg})$ & $0.279 / 0.100$ & $0.337 / 0.018^{*}$ \\
DBP $(\mathrm{mmHg})$ & $0.259 / \mathrm{NS}$ & $0.288 / 0.088$ \\
Creatinine $(\mu \mathrm{mol} / \mathrm{l})$ & $0.369 / 0.027^{*}$ & $0.392 / 0.018^{*}$ \\
FG $(\mathrm{mmol} / \mathrm{l})$ & $0.094 / \mathrm{NS}$ & $0.096 / \mathrm{NS}^{*}$ \\
FI (pmol/l) & $-0.515 / 0.001^{*}$ & $-0.392 / 0.018^{*}$ \\
HOMA-IR & $-0.423 / 0.010^{*}$ & $-0.316 / 0.060$ \\
FFA (mmol/l) & $0.268 / \mathrm{NS}$ & $0.381 / 0.022^{*}$ \\
Cholesterol (mmol/l) & $-0.216 / \mathrm{NS}$ & $-0.104 / \mathrm{NS}$ \\
TG (mmol/l) & $-0.159 / \mathrm{NS}$ & $-0.106 / \mathrm{NS}$ \\
Total adiponectin & $0.910 / 0.000^{*}$ & - \\
(mg/l) & & \\
HMW adiponectin & - & $0.910 / 0.000^{*}$ \\
(mg/l) & & $0.214 / \mathrm{NS}$ \\
CRP (mg/l) & $0.228 / \mathrm{NS}$ & \\
\hline
\end{tabular}

$\mathrm{BMI}$, body mass index; CRP, C reactive protein; DBP, diastolic blood pressure; FFA, free fatty acids; $F G$, fasting glucose; $F$, fasting insulin; HOMA-IR, Homeostasis model assessment of insulin resistance; SBP, systolic blood pressure; TG, triglycerides. $r / P$ values are given. NS indicates $P>0.1$ *Significant correlation as assessed by Spearman's correlation method.

the two parameters were included in the model (Table 3). Furthermore, PE remained a significant independent predictor of both HMW adiponectin and total adiponectin in multivariate analyses (Table 3). After controlling for PE, fasting insulin predicted HMW adiponectin but not total adiponectin (Table 3). Moreover, the association between HMW adiponectin and total adiponectin on one hand and creatinine on the other hand, was lost after controlling for PE (Table 3).

\section{Discussion}

In the current study, we demonstrate that maternal serum concentrations of HMW adiponectin are significantly increased in PE patients when compared with healthy gestational age-matched controls. Using a different experimental approach, a similar finding has recently been obtained by Takemura et al. (28). The authors demonstrate that in their hands median HMW adiponectin levels are $11.2 \mathrm{mg} / \mathrm{l}$ in the PE patients when compared with $6.8 \mathrm{mg} / \mathrm{l}$ in the controls using an ELISA kit from Daiichi Pure Chemicals (Tokyo, Japan) (28). In addition, we demonstrate that total circulating adiponectin is significantly increased in PE patients in accordance with previous studies (19-23). Furthermore, we show for the first time in pregnant women that HMW adiponectin is positively correlated with creatinine and negatively associated with markers of insulin resistance (fasting insulin and HOMA-IR) in univariate analyses. A negative correlation between HMW adiponectin and fasting insulin is also demonstrated when the subgroups (control, PE) are studied separately. Moreover, we are the first to demonstrate that fasting 
Table 3 Dependent variable: high-molecular-weight (HMW) adiponectin or total adiponectin.

\begin{tabular}{llcr}
\hline Model & Independent variable & HMW adiponectin $\beta / P$ & Total adiponectin $\beta / P$ \\
\hline Model 1 & FI & $-0.414 / 0.007^{*}$ & $-0.313 / 0.041^{*}$ \\
& Creatinine & $0.353 / 0.019^{*}$ & $0.411 / 0.008^{*}$ \\
Model 2 & FI & $-0.320 / 0.031^{*}$ & $-0.236 / \mathrm{NS}$ \\
& Creatinine & $0.120 / \mathrm{NS}$ & $0.156 / \mathrm{NS}$ \\
& BMI & $-0.117 / \mathrm{NS}$ & $-0.063 / \mathrm{NS}$ \\
Model 3 & FI & $0.497 / 0.004^{*}$ & $0.514 / 0.003^{*}$ \\
& Creatinine & $-0.359 / 0.010^{*}$ & $-0.261 / 0.059$ \\
& FFA & $0.136 / \mathrm{NS}$ & $0.152 / \mathrm{NS}$ \\
& PE & $-0.081 / \mathrm{NS}$ & $-0.001 / \mathrm{NS}$ \\
& & $0.494 / 0.006^{*}$ & $0.495 / 0.006^{*}$ \\
\hline
\end{tabular}

Multivariate linear regression analyses in all subjects $(n=36)$ between $\mathrm{HMW}$ adiponectin or total adiponectin (dependent variable) and FI and creatinine (Model 1 ) adjusted for BMI and PE (Model 2), as well as adjusted for FFA and PE (Model 3). FFA, free fatty acids; FI, fasting insulin. $\beta$-coefficients/P values are given. NS indicates $P>0.1$. * Significant correlation.

insulin remains an independent predictor of circulating HMW adiponectin also after adjustment for serum creatinine and PE in pregnant patients. In contrast, the association between total adiponectin and fasting insulin is lost after controlling for PE. These results indicate that the close relationship between HMW adiponectin and insulin sensitivity is present not only in controls but also in PE patients and that HMW adiponectin might be a better marker of insulin sensitivity when compared with total adiponectin.

Upregulation of HMW adiponectin in PE appears paradoxical since this pregnancy-associated complication is linked to increased risk of cardiovascular and metabolic diseases and HMW adiponectin is regarded as a protective factor for both conditions. Thus, changes in serum HMW adiponectin but not total adiponectin are associated with improvement of hepatic insulin sensitivity after thiazolidinedione treatment $(17,29)$. Another report suggests that only HMW adiponectin induces activation of AMP kinase in muscle (30). Furthermore, HMW adiponectin suppresses apoptosis in cultured endothelial cells in vitro (18). Most recently, Torigoe et al. have shown that serum levels of HMW adiponectin predict endothelial dysfunction in young men before any overt vascular disease has occurred (31). Taking these studies into consideration, increased HMW adiponectin concentrations in PE could be part of a physiological feedback improving insulin sensitivity and vascular function in PE.

It remains unclear by which mechanisms HMW adiponectin and total adiponectin are upregulated in PE. For total adiponectin, it has been postulated that elevated secretion of this adipokine from fat might contribute to increased serum levels found in PE (19). In contrast to this hypothesis, a significant difference in adiponectin mRNA expression could not be demonstrated in adipose tissue of PE patients when compared with healthy controls (20). Furthermore, adipokines upregulated in PE including TNF- $\alpha$ and IL- 6 have recently been shown by our group to significantly downregulate adiponectin in fat (32-34). However, it is interesting to note in this context that serum creatinine is significantly and positively correlated to HMW adiponectin in the current study independent of fasting insulin levels. Furthermore, we show that serum creatinine is significantly increased in PE patients when compared with gestational age-matched controls. Taking these data into consideration, it appears plausible that mild renal dysfunction seen as a consequence of $\mathrm{PE}$ at least in part contributes to increased circulating HMW adiponectin in this pregnancy-associated disease. This hypothesis is further supported by reports convincingly demonstrating that renal excretion is a physiologically important route of adiponectin clearance with increased levels of this adipokine found in end-stage renal disease $(35,36)$.

Taken together, we present evidence that maternal HMW adiponectin levels are significantly increased in PE and positively associated with markers of insulin sensitivity and renal dysfunction. Our data support the hypotheses that HMW adiponectin might be part of a physiological feedback mechanism improving insulin sensitivity and cardiovascular health in PE and that renal excretion is a physiological route of HMW adiponectin clearance.

\section{Acknowledgements}

This study was supported by a grant from the Deutsche Forschungsgemeinschaft (DFG), KFO 152: 'Atherobesity', project FA476/4-1 (TP 4) to M F, project BL833/1-1 (TP3) to M B, and the IZKF Leipzig to M F (Project B25).

\section{References}

1 Sibai B, Dekker G \& Kupferminc M. Pre-eclampsia. Lancet 2005 365 785-799.

2 Stepan H, Geide A \& Faber R. Soluble fms-like tyrosine kinase 1. New England Journal of Medicine $20043512241-2242$.

3 Stepan H \& Faber R. Elevated sFlt1 level and preeclampsia with parvovirus-induced hydrops. New England Journal of Medicine 2006 354 1857-1858.

4 Stepan H \& Walther T. Questionable role of the angiotensin II receptor subtype 1 autoantibody in the pathogenesis of preeclampsia. Hypertension $2007 \mathbf{5 0}$ e3. 
5 Stepan H, Unversucht A, Wessel N \& Faber R. Predictive value of maternal angiogenic factors in second trimester pregnancies with abnormal uterine perfusion. Hypertension $2007 \mathbf{4 9} 818-824$.

6 Poston L. Leptin and preeclampsia. Seminars in Reproductive Medicine 200220 131-138.

7 Tommaselli GA, Pighetti M, Nasti A, D’Elia A, Guida M, Di Carlo C, Bifulco G \& Nappi C. Serum leptin levels and uterine Doppler flow velocimetry at 20 weeks' gestation as markers for the development of pre-eclampsia. Gynecological Endocrinology 200419 160-165.

8 Conrad KP, Miles TM \& Benyo DF. Circulating levels of immunoreactive cytokines in women with preeclampsia. American Journal of Reproductive Immunology 199840 102-111.

9 LaMarca BB, Bennett WA, Alexander BT, Cockrell K \& Granger JP. Hypertension produced by reductions in uterine perfusion in the pregnant rat: role of tumor necrosis factor-alpha. Hypertension $2005461022-1025$.

10 Freeman DJ, McManus F, Brown EA, Cherry L, Norrie J, Ramsay JE, Clark P, Walker ID, Sattar N \& Greer IA. Short- and long-term changes in plasma inflammatory markers associated with preeclampsia. Hypertension $2004 \mathbf{4 4} 708-714$.

11 Maeda N, Shimomura I, Kishida K, Nishizawa H, Matsuda M, Nagaretani H, Furuyama N, Kondo H, Takahashi M, Arita Y, Komuro R, Ouchi N, Kihara S, Tochino Y, Okutomi K, Horie M, Takeda S, Aoyama T, Funahashi T \& Matsuzawa Y. Diet-induced insulin resistance in mice lacking adiponectin/ACRP30. Nature Medicine 20028 731-737.

12 Kubota N, Terauchi Y, Yamauchi T, Kubota T, Moroi M, Matsui J, Eto K, Yamashita T, Kamon J, Satoh H, Yano W, Froguel P, Nagai R, Kimura S, Kadowaki T \& Noda T. Disruption of adiponectin causes insulin resistance and neointimal formation. Journal of Biological Chemistry 2002277 25863-25866.

13 Matsuda M, Shimomura I, Sata M, Arita Y, Nishida M, Maeda N, Kumada M, Okamoto Y, Nagaretani H, Nishizawa H, Kishida K, Komuro R, Ouchi N, Kihara S, Nagai R, Funahashi T \& Matsuzawa Y. Role of adiponectin in preventing vascular stenosis. The missing link of adipo-vascular axis. Journal of Biological Chemistry 2002277 37487-37491.

14 Yamauchi T, Kamon J, Waki H, Imai Y, Shimozawa N, Hioki K, Uchida S, Ito Y, Matsui J, Eto K, Komeda K, Tsunoda M, Murakami K, Ohnishi Y, Yamamura K, Ueyama Y, Froguel P, Kimura S, Nagai R \& Kadowaki T. Globular adiponectin protected ob/ob mice from diabetes and apoE deficient mice from atherosclerosis. Journal of Biological Chemistry 2003278 2461-2468.

15 Kralisch S, Klein J, Bluher M, Paschke R, Stumvoll M \& Fasshauer M. Therapeutic perspectives of adipocytokines. Expert Opinion on Pharmacotherapy 20056 863-872.

16 Sattar N, Wannamethee G, Sarwar N, Tchernova J, Cherry L, Wallace AM, Danesh J \& Whincup PH. Adiponectin and coronary heart disease: a prospective study and meta-analysis. Circulation $2006114623-629$.

17 Pajvani UB, Hawkins M, Combs TP, Rajala MW, Doebber T, Berger JP, Wagner JA, Wu M, Knopps A, Xiang AH, Utzschneider KM, Kahn SE, Olefsky JM, Buchanan TA \& Scherer PE. Complex distribution, not absolute amount of adiponectin, correlates with thiazolidinedione-mediated improvement in insulin sensitivity. Journal of Biological Chemistry 2004279 12152-12162.

18 Kobayashi H, Ouchi N, Kihara S, Walsh K, Kumada M, Abe Y, Funahashi T \& Matsuzawa Y. Selective suppression of endothelial cell apoptosis by the high molecular weight form of adiponectin. Circulation Research 200494 e27-e31.

19 Ramsay JE, Jamieson N, Greer IA \& Sattar N. Paradoxical elevation in adiponectin concentrations in women with preeclampsia. Hypertension $2003 \mathbf{4 2} 891-894$.

20 Haugen F, Ranheim T, Harsem NK, Lips E, Staff AC \& Drevon CA. Increased plasma levels of adipokines in preeclampsia: relationship to placenta and adipose tissue gene expression. American Journal of Physiology, Endocrinology and Metabolism 2006290 E326-E333.

21 Kajantie E, Kaaja R, Ylikorkala O, Andersson S \& Laivuori H. Adiponectin concentrations in maternal serum: elevated in preeclampsia but unrelated to insulin sensitivity. Journal of the Society for Gynecologic Investigation 200512 433-439.
22 Hendler I, Blackwell SC, Mehta SH, Whitty JE, Russell E, Sorokin Y \& Cotton DB. The levels of leptin, adiponectin, and resistin in normal weight, overweight, and obese pregnant women with and without preeclampsia. American Journal of Obstetrics and Gynecology 2005193 979-983.

23 Naruse K, Yamasaki M, Umekage H, Sado T, Sakamoto Y \& Morikawa H. Peripheral blood concentrations of adiponectin, an adipocyte-specific plasma protein, in normal pregnancy and preeclampsia. Journal of Reproductive Immunology 200565 65-75.

24 D'Anna R, Baviera G, Corrado F, Giordano D, Di Benedetto A \& Jasonni VM. Plasma adiponectin concentration in early pregnancy and subsequent risk of hypertensive disorders. Obstetrics and Gynecology 2005106 340-344.

25 Nakano Y, Tajima S, Yoshimi A, Akiyama H, Tsushima M, Tanioka T, Negoro T, Tomita M \& Tobe T. A novel enzyme-linked immunosorbent assay specific for high-molecular-weight adiponectin. Journal of Lipid Research 200647 1572-1582.

26 National High Blood Pressure Education Program Working Group on High Blood Pressure in Pregnancy. Report of the National High Blood Pressure Education Program Working Group on High Blood Pressure in Pregnancy. American Journal of Obstetrics and Gynecology 2000183 S1-S22.

27 Fasshauer M, Bluher M, Stumvoll M, Tonessen P, Faber R \& Stepan H. Differential regulation of visfatin and adiponectin in pregnancies with normal and abnormal placental function. Clinical Endocrinology $2007 \mathbf{6 6} 434-439$.

28 Takemura Y, Osuga Y, Koga K, Tajima T, Hirota Y, Hirata T, Morimoto C, Harada M, Yano T \& Taketani Y. Selective increase in high molecular weight adiponectin concentration in serum of women with preeclampsia. Journal of Reproductive Immunology 200773 60-65.

29 Tonelli J, Li W, Kishore P, Pajvani UB, Kwon E, Weaver C, Scherer PE \& Hawkins M. Mechanisms of early insulin-sensitizing effects of thiazolidinediones in type 2 diabetes. Diabetes 200453 1621-1629.

30 Waki H, Yamauchi T, Kamon J, Ito Y, Uchida S, Kita S, Hara K, Hada Y, Vasseur F, Froguel P, Kimura S, Nagai R \& Kadowaki T. Impaired multimerization of human adiponectin mutants associated with diabetes. Molecular structure and multimer formation of adiponectin. Journal of Biological Chemistry 2003278 40352-40363.

31 Torigoe M, Matsui H, Ogawa Y, Murakami H, Murakami R, Cheng XW, Numaguchi Y, Murohara T \& Okumura K. Impact of the high-molecular-weight form of adiponectin on endothelial function in healthy young men. Clinical Endocrinology 200767 276-281.

32 Fasshauer M, Klein J, Neumann S, Eszlinger M \& Paschke R. Hormonal regulation of adiponectin gene expression in 3T3-L1 adipocytes. Biochemical and Biophysical Research Communications $20022901084-1089$.

33 Fasshauer M, Klein J, Neumann S, Eszlinger M \& Paschke R. Adiponectin gene expression is inhibited by beta-adrenergic stimulation via protein kinase $\mathrm{A}$ in 3T3-L1 adipocytes. FEBS Letters $2001 \mathbf{5 0 7} 142-146$.

34 Fasshauer M, Kralisch S, Klier M, Lossner U, Bluher M, Klein J \& Paschke R. Adiponectin gene expression and secretion is inhibited by interleukin-6 in 3T3-L1 adipocytes. Biochemical and Biophysical Research Communications 2003301 1045-1050.

35 Chudek J, Adamczak M, Karkoszka H, Budzinski G, Ignacy W, Funahashi T, Matsuzawa Y, Cierpka L, Kokot F \& Wiecek A. Plasma adiponectin concentration before and after successful kidney transplantation. Transplantation Proceedings 200335 2186-2189.

36 Zoccali C, Mallamaci F, Tripepi G, Benedetto FA, Cutrupi S, Parlongo S, Malatino LS, Bonanno G, Seminara G, Rapisarda F, Fatuzzo P, Buemi M, Nicocia G, Tanaka S, Ouchi N, Kihara S, Funahashi T \& Matsuzawa Y. Adiponectin, metabolic risk factors, and cardiovascular events among patients with end-stage renal disease. Journal of the American Society of Nephrology 200213 134-141.

Received 7 November 2007

Accepted 12 November 2007 